\title{
Reducción de la Anemia en Gestantes a través de una Intervención con Base Comunitaria
}

\author{
LOURDES ELÍAS, GLADYS VÍA, MARIZOL RODRÍGUEZ, IRIS TINOCO \\ Organismo No Gubernamental "Fomento de la Vida" (FOVIDA)
}

\begin{abstract}
RESUMEN
OBJETIVO: Evaluar el impacto de una intervención preventivo-promocional, basada en Ia comunidad, para mejorar el conocimiento y el consumo de hierro de las mujeres gestantes con anemia. MÉTODOS: EI estudio se realizó en las zonas urbano marginales del Cono Sur de Lima - Perú; se encuestó 76 mujeres anémicas en el $2^{\circ}$ trimestre de embarazo, predominantemente jovenes $(50 \%, 17 \%$ adolescentes). La intervención se caracterizó por la participación de las mujeres organizadas en la promoción de la salud de la mujer, a través de la suplementación y monitorización del consumo de hierro, por el conocimiento del perfil sociocultural de las gestantes anémicas y la realización de una intervención en Información, Educación y Comunicación (IEC). RESULTADOS: Existe un alto porcentaje de mujeres con antecedente de aborto (23\%). Algo más de la mitad (51\%) de las madres se informaron a través de las promotoras de salud sobre la utilidad del consumo de hierro. Se recuperaron de la ancmia $55 \%$ de las gestantes, obteniéndose respuesta terapéutica en $45 \%$ y fracaso en $17 \%$. CONCLUSIÓN: Se pudo mejorar después de la intervención en IEC la práctica consciente del consumo de hierro en $97 \%$. El seguimiento periódico y la orientación individual transmitida de manera permanente y adecuada fueron trascendentales.
\end{abstract}

Palabras claves: Anemia Hipocrómica; Embarazo; Educación en Salud; Encuestas Nutricionales.

\section{REDUCTION OF ANEMIA IN PREGNANT WOMEN THROUGH A COMMUNITY- BASED APPROACH SUMMARY}

OBJECTIVE: To study the impact of a community-based preventive-promotional approach to improve the knowledge and attitudes of pregnant women with anemia toward the iron intake. METHODS: 76 pregnant women with anemia at II trimester of gestation, mainly young females from South marginal-suburban zones at Lima-Pcru, were studied. This research was lied in health promoter women, the knowledge of the social-cultural profile of the pregnant, and an Informative, Educational and Communicative (IEC) approach. RESULTS: There were many women (23\%) with history of abort gestation, and more than a half of mothers $(51 \%)$ have learned about iron intake and its benefits by health promoters. $55 \%$ anemic women were healthy at the endpoint of the study. The therapeutic responses and failures were $45 \%$ and $17 \%$, respectively. CONCLUSIONS: After the IEC approach, informed iron-intake attitude increased by $97 \%$. Adequate persistent follow-up and personal arrangement was cardinal to improve the outcomes.

Key words: Anemia, Hypochromic; Pregnancy; Health Education; Nutrition Surveys.

\footnotetext{
Correspondencia:

Lic. Lourdes Elías Peñafiel.

Fomento de la Vida.

Av. Javier Prado Oeste 109. Lima 17 - Perix.

E-mail: lourdes@fovida.org.pe
} 


\section{INTRODUCCIÓN}

La anemia en el ámbito poblacional es generalmente causada por deficiencia de hierro en la dieta y afecta a nivel mundial a cerca de 2000 millones de personas ('), lo que la que convierte en la deficiencia nutricional más frecuente. En países en desarrolio, la prevalencia de la anemia promedia el $56 \%$, con valores entre 35 y $75 \%$ entre diferentes regiones del mundo. Situacion opuesta a la de paśses desarrollados que tienen prevalencia promedio de $18 \%$. América Latina tiene $39 \%$ de gestantes con niveles bajos de hemoglobina, que se distribuyen con 37\% en América Central y del Sury $52 \%$ en el Caribe ( ${ }^{1}$ ). La última encuesta demográfica y de salud a nivel nacional (ENDES 1996) ( ${ }^{2}$ ) mostró una prevalencia global de $36 \%$ en el Perú, con relativamente poca diferencia entre regiones geográficas.

En gestantes de Lima, la prevalencia de anemia en 1993 fue $54,6 \%\left({ }^{3}\right)$, mientras que para mujeres no gestantes de distintos estratos socioeconómicos de Lima Metropolitana ha estado en el rango de $16,8 \%$ (alto) a 29,8\% (bajo) ( ${ }^{4}$ ). En otras regiones del Perú, la prevalencia en mujeres en edad fértil llega a $83,5 \%$ (Ica) y 74,7\% (Ayacucho) $\left({ }^{4}\right)$. La anemia es una de las causas indirectas que actúan sobre la morbi-mortalidad materna, por lo que es considerada problema prioritario de salud materna por parte de las políticas de salud (').

En áreas libres de malaria o uncinariasis, los principales factores determinantes son el consumo de alimentos con hierro de baja biodisponibilidad y estados fisiológicos como crecimiento rápido en la infancia y gestación, que implican requerimientos incrementados prácticamente imposibles de cubrir con la dieta habitual. Si la dieta contiene hierro con biodisponibilidad promedio de 5\% (baja), la gestante requerirá tanto como $60 \mathrm{mg} /$ día de hierro en la dieta ( $\left.{ }^{5}\right)$. En prácticamente todos los contextos, las gestantes necesitan suplementos de hierro desde el $4^{\circ}$ mes de gestación, los que deben continuar en el puerperio durante dos o más meses $\left({ }^{6}\right)$.

Las consecuencias de la anemia son la mortalidad materna por complicaciones obstétricas, que llega a ser ocho veces mayor cuando la hemoglobina es menor de $8 \mathrm{~g} / \mathrm{dL}$. También lo son la prematuridad, peso bajo al nacer y la mortalidad perinatal ("). La anemia reduce la productividad en el trabajo físico, por lo que perpetúa la pobreza al reducir los ingresos económicos (').

En zonas con bajas coberturas de parto institucional y de control prenatal, se requiere de programas basados en la comunidad que se apoyen en las mujeres organizadas para aumentar la cobertura de la suplementación con hierro de las gestantes. El objetivo de este trabajo fue evaluar el impacto de una intervención basada en la comunidad para mejorar el conocimiento y el consumo de hierro en gestantes.

\section{MATERIAL Y MÉTODOS}

\section{Diseño}

Es un estudio longitudinal prospectivo de tipo cuasiexperimental. Duración nueve meses.

\section{Universo}

Estuvo conformado por 99 gestantes de las comunidades de Delicias de Villa (Chomillos) y Nueva Esperanza (Villa María del Triunfo), con menos de 26 semanas y sin antecedentes de uso de suplementos de hierro.

Fueron reclutadas por barrido casa por casa realizado por las promotoras de salud de las respectivas comunidades.

\section{Muestra}

Fue intencionada, por conveniencia, no probabilística y la definimos como la totalidad de mujeres anémicas $(<11 \mathrm{~g}$ ) dL), abarcando 76 mujeres.

\section{Estudio Sociocultural}

Nos planteamos trabajar en el recojo de información cuantitativa y cualitativa, considerando que son complementarias y juntas nos ofrecen mayor y mejor información $\left(^{8}\right)$.

Se adaptó la metodología de asesońa rápida (RAP) $\left({ }^{9}\right)$ elaborando una encuesta, guías para entrevista y guías para observación.

Los instrumentos de recolección fueron:

- Encuesta de Conocimientos y Prácticas, al 100\% de las gestantes anémicas de ambas comunidades. Constaba de cuatro secciones: a) información general, b) consumo y percepción sobre el hierro, c) educación en salud, y d) comunicación en salud, con preguntas precodificadas en su mayoría y algunas preguntas abiertas.

- Entrevistas a profundidad, aplicadas a una submuestra de las mismas gestantes determinada al dzar, a quienes se les visitó en sus domicilios, con la finalidad de complementar la información obtenida en las encuestas para lograr un panorama más completo de la realidad de las mismas. 
Ensayo Clínico Abierto No Controlado de Suplementación de Hierro más Estrategia de IEC en Gestantes Anémicas

Análisis de Laboratorio: El cambio en el nivel de hierro fue medido en todas las gestantes mediante el valor de hemoglobina, utilizando el método de la cianmetahemoglobina, tomando en cuenta el punto de corte de $11 \mathrm{~g} / \mathrm{dL}$. Se realizaron estas pruebas en los laboratorios de los servicios de salud de cada comunidad. El intervalo del control de hernoglobina inicial y final fue 15 semanas.

Suplementación de Hierro: La suplementación de hierro tuvo una duración de 15 semanas consecutivas, por día se administró una tableta de sulfato ferroso (Laboratorio COFANA) de $300 \mathrm{mg}$ equivalente a $60 \mathrm{mg}$ de hierro elemental. Éstas no contenían ácido fólico ni vitamina $C$.

Durante las visitas domiciliarias, las promotoras usaban como instrumento una ficha de seguimiento que verificaba el consumo diario de las tabletas, la presencia de efectos colaterales y su asistencia al control prenatal. Se distribuía 20 tabletas por visita y a la siguiente vez les solicitaban a las gestantes los blisters vacíos.

Les explicaban y distribuían material impreso con las siguientes recomendaciones:

- Tomar las tabletas de preferencia con limonada, jugo de naranja o de papaya, o con agua sola.

- No tomar café, té o mates.

- Tomarlas una hora antes o después de los alimentos.

- Preservar las tabletas del sol y la humedad.

\section{Participación Comunal}

El reclutamiento de las gestantes, la distribución y supervisión de la ingesta de las tabletas de hieno fue realizado por las promotoras de salud. Esta intervención se incluyó en el Sistema de Vigilancia de la Salud Materna, detectando oportunamente los factores de riesgo en la gestación y puerperio, y refiriéndolas a los servicios de salud. Ésta es una línea de intervención del proyecto Salud Mujer de FOVIDA.

Durante las visitas domiciliarias periódicas, las promotoras informaban y orientaban a las gestantes con mensajes básicos y sencillos respecto a la anemia y su prevención.
El número de promotoras participantes en ambas comunidades fue veintiuno.

\section{Estrategia de Información, Educación y Comuni-} cación (IEC)

La propuesta de IEC en el control de la anemia estuvo confomada por:

- Diseño de acciones educativas enfocadas a mejorar las prácticas de salud de las gestantes: Sociodrama, cuyo guión fue preparado y realizado por las promotoras.

- Diseño y elaboración de materiales educativos para gestantes: Juego educativo "El fierrito", tríptico "Nutrición durante el Embarazo".

- Diseño de acciones de difusión y comunicación basados en las necesidades de información y en la realidad local, dirigido a 76 familias: Feria de Salud y Pasacalle que combinaba elementos de comunicación, educación en salud, recreación, festividad y esparcimiento para las gestantes en una sola actividad, donde también participaban sus esposos, hijos y otros familiares. Incorporaba la degustación de platos preparados a base de sangrecita, difusión del recetario "La Canasta de Recetas para Embarazadas", polos estampados con los personajes descritos (sangrecita y fierrito), afiche diseñado por las promotoras "Prevenir la Anemia con Sangrecita y Fierrito", murales pintados en los Servicios de Salud con el tema central de la prevención de la anemia y promoción del consumo de hierro y sociodrama realizado por las promotoras de salud con el mismo tema central.

- Diseño y ejecución de talleres de comunicación en salud con promotoras y gestantes anémicas: Focus Group.

\section{RESULTADOS}

Los resultados obtenidos en el Estudio Sociocultural, nos permitió identificar aspectos como la información que tenían las gestantes sobre la anemia, sus hábitos, costumbres y consumo de alimentos.

También se pudo conocer el tipo de relaciones interpersonales que presentan al interior de su familia y el mundo interno de la gestante (como es su autoestima, su autopercepción y sus perspectivas personales).

Las encuestas mostraron que alrededor de la mitad de las madres anémicas eran migrantes de primera generación, 
procedentes del Norte (Piura, Cajamarca) y del Sur (Apurímac, Cuzco), habiendo llegado a Lima a corta edad.

Se caracterizaban por ser predominantemente jóvenes (50\%), donde el $17 \%$ eran adolescentes (Tabla $N^{\circ} 1$ ).

Tabla N ${ }^{\circ}$ 1.- Gestantes estratificadas según edad.

\begin{tabular}{cc}
\hline $\begin{array}{c}\text { Edad } \\
\text { (años) }\end{array}$ & Porcentaje \\
\hline $15-19$ & $17 \%$ \\
$20-24$ & $33 \%$ \\
$25-34$ & $32 \%$ \\
$>35$ & $18 \%$ \\
\hline
\end{tabular}

El grado de insurucción fue primaria en el $25 \%$ de gestantes, secundaria en el $66 \%$ y superior en el $9 \%$. Se encontraban en unión de convivencia $60 \%$, declararon ser casadas $34 \%$ y sólo $6 \%$ solteras. La mayoría de las gestantes tenían más de dos hijos ( 2 a 3 hijos: 50\%, 4 o más hijos: 16\%) y sólo 34\% con un hijo. Se evidenció el deseo de limitar el numero de hijos, si es que eso se puede inferir del elevado porcentaje de embarazos no deseados (44\%) así como en el número de mujeres con antecedente de un aborto (23\%).

Las gestantes tienen hijos de corta edad, el 68\% de ellas tienen hijos menores de tres años.

El nivel económico de las familias de las gestantes no cubre la canasta básica de alimentos en un $60 \%\left({ }^{10}\right)$. Las condiciones de trabajo son difíciles y las remuneraciones obtenidas no conresponden al tiempo de trabajo realizado, el $8 \%$ de las gestantes se encuentran fuera de su hogar en el comercio ambulatorio (5\%) o empleadas (3\%, ayudante de cocina y lavado de ropa); en general, empleos de bajo nivel de ingresos, significando que el $92 \%$ se encuentra dependiendo económicamente de sus esposos. Ellos no cuentan con ingresos permanentes frente a sus necesidades básicas. Las labores principales que realizan los esposos son albañilería, cobradores de microbus, vigilantes, etc.

La gestación no parece lograr cambios en la rutina diaria y de trabajo de la mujer. El $93 \%$ de las mujeres entrevistadas a profundidad mostraron poca preocupación por su salud y Ia anemia, subordinando el cuidado de su salud ante el cuidado de la salud del esposo y de los hijos. También mostraron baja autoestima (Tabla $\mathrm{N}^{\circ}$ 2).

Las entrevistas en profundidad permitieron identificar que el escaso consumo de suplementos de hierro por las gestantes antes de la intervención estaba asociado con falta de consejería por parte del personal a cargo del control prenatal. Hubo una escasa o nula difusión sobre la oferta de suplementos para gestantes en los establecimientos de salud. Por otro lado, el abastecimiento de insumos (sales de hieno) era inadecuado e insuficiente, estando focalizado en quienes tenían anemia severa y sólo durante un mes.

En relación al conocimiento de dietas adecuadas el $74 \%$ manifestaron desinformación, se observó también la presencia de fuertes mitos tradicionales y culturales $(80 \%)$, como "fatalismo" (palidez en la gestante) que significa para ellas que el niño se está alimentando bien.

Para desarrollar en las gestantes la necesidad consciente de consumir hierro como medida de tratamiento o prevención frente a la anemia, fue muy importance su propia participación así como también de las familias en las actividades informativas y de comunicación.

Tabla $N^{\circ}$ 2.- Características culturales de las gestantes anémicas.

\section{Baja autoestima}

- Poca preocupación por su salud y por la anemia

- Subordina su salud, su autocuidado ante la pareja y los hijos

- Embarazos no deseados

Malos hábitos alimenticios de la gestante \%

- Presencia de fuertes mitos tradicionales y culturales

- Desinformación sobre dietas y recetas adecuadas 
Esta participación de la población no fue pasiva sino con responsabilidades en cuanto a su salud y bienestar propio y colectivo $\left({ }^{11}\right)$.

En las visitas domiciliarias se establecía una comunicación interpersonal, se emitieron consejos prácticos para desalentar el consumo de inhibidores de la absorción de hierro junto con los alimentos (café, infusiones de hierbas) y fomentar el consumo de facilitadores de la absorción de hierro no heme-como vitamina $\mathrm{C}\left({ }^{12}\right)$ (jugo de naranja, limonada, papaya). Los efectos colaterales fueron manejados a través de la emisión de mensajes por parte de las promotoras, como división de la dosis o tomar las tabletas al acostarse.

Las promotoras de salud tuvieron una elevada credibilidad como fuente de orientación -más asequibles y cálidas que el personal de salud- y de suministro de hierro. Esto favoreció la relación de empatía entre las promotoras y las madres, evidenciándose un cambio en las prácticas del consumo de hierro y la valoración de esta acción.

Las encuestas revelaron que algo más de la mitad (51\%) de las madres se informaron a través de las promotoras de salud sobre la anemia y la necesidad de consumir suplementos de hienro. También reflejaron cambios en el nivel de consumo efectivo del mismo ( $97 \%$ de las gestantes consumieron en forma regular las 105 tabletas planeadas) (Tabla $\mathrm{N}^{\circ} 3$ ).

Tabla $N^{\circ}$ 3.- Cambios en el nivel de información y en el consumo regular de hierro en las gestantes.

\begin{tabular}{lcc}
\hline \multicolumn{1}{c}{ Parámetro observado } & \multicolumn{2}{c}{ Intervención (\%) } \\
\cline { 2 - 3 } & antes & después \\
\hline Nivel de información sobre hierro & 0 & 97 \\
Nivel de consumo de hierro & 51 & 97 \\
\hline
\end{tabular}

En las campañas de información, educación y comunicación de ambas comunidades se dieron mensajes básicos como: "sangrecita y fienito juntos contra la anemia", "embarazo sin anemia, embarazo feliz", "la anemia es muy frecuente en el embarazo, por eso debes comer balanceado y complementarlo con hierro".
Los símbolos o personajes fueron dos dibujos cuyos bocetos fueron elaborados en un taller con gestantes anémicas. La "Sangrecita" representa una gota de sangre animal usada en la alimentación. El grupo focal de gestantes recomendó que fuera representada como una figura antropomorfizada de sexo femenino, atractiva, bien vestida. El "Fierrito" debía ser una figura masculina en la forma de una cápsula de hierro. Ambos personajes deberían ser representados como una pareja armoniosa. Estos fueron los símbolos de imagen global de las campañas.

En dichas campañas se trabajaron factores determinantes en la conducta de la gestante como: la motivación, la información, la autoestima, la decision y la destreza. Se adecuaron para ello un enfoque de intervención educativa en nutrición $\left({ }^{13.14}\right)$. Las campañas también combinaban el uso de diferentes medios y eran de corta duración $\left({ }^{15}\right)$.

Las intervenciones de IEC fueron evaluadas por las gestantes anémicas: el 96,2\% manifestó que el recetario era fácil de comprender, mientras que el 30,2\% recordaba la idea central de las campañas: "consumo de sangrecita y fierrito".

Los mensajes educativos llegaron a tener éxito pues se tomaron en cuenta las motivaciones reales del grupo objetivo.

En lo referente a los materiales producidos para las ferias, cumplieron con su finalidad el $93 \%$ de ellos y tuvieron la siguiente jerarquización según la aceptación de las madres: el recetario ocupa el primer lugar (48\%), el juego educativo "El Fierrito" 29\%, y otros como volantes, afiches y polos estampados representan el 23\% (Tabla No 4). Este orden sugiere una preferencia por los materiales que tienen una utilidad inmediata: recomendaciones sobre la dieta (recetario) y juego con información educativa.

En cuanto a los cambios en la dieta, los testimonios de las madres recogidos en evaluaciones y en contactos con las promotoras revelaron que éstas empleaban las recetas transmitidas y que gustaban de las preparaciones basadas en sangre animal.

La eficacia de la suplementación con hierro en tratar la anemia fue confirmada al realizar el control de hemoglobina inicial ( $77 \%$ ) y final ( $55 \%$ ) al final de las 15 semanas. El $45 \%$ presentó respuesta terapéutica y el $17 \%$ fracaso terapéutico, cuando se consideró el porcentaje de gestantes que incrementaron $\mathrm{I} \mathrm{gr} / \mathrm{dL}$ contra aquellas en donde los niveles permanecieron igual o disminuyeron, respectivamente. 
Tabla $N^{\circ}$ 4.- Resultados de la evaluación de intervenciones de información, educación y comunicación (IEC) realizada por las gestantes anérnicas.

\begin{tabular}{lc}
\hline Cumplieron su finalidad & $93 \%$ \\
$\begin{array}{l}\text { Recetario fácil de comprender } \\
\text { Recordaba la idea central "consumo de }\end{array}$ & $96 \%$ \\
sangrecita y fierrito" & $30 \%$ \\
$\begin{array}{l}\text { Preferencia de las madres por los } \\
\text { materiales producidos }\end{array}$ \\
- Recetario & \\
- Juego educativo "El Fierrito" & $48 \%$ \\
- Otros & $29 \%$ \\
\hline
\end{tabular}

\section{DISCUSIÓN}

La anemia afecta a mujeres de clase media y baja, mientras que las del estrato socioeconómico alto están protegidas, presumiblemente por una mejor calidad de la dieta y acceso a servicios preventivos y curativos $\left({ }^{12}\right)$. Según una experiencia en comedores populares donde acuden mujeres pobres anémicas a consumir alimentos, se logró generar una percepción acerca de los alimentos fuente de hierro, la revalorización de la mujer y su autoafirmación con un enfoque educativo-nutricional $\left({ }^{14}\right)$.

La prioridad para suplementación son los niños y las gestantes porque tienen mayor prevalencia de anemia $\left({ }^{16}\right)$, por los beneficios de las actividades de control desde el punto de vista de la salud pública y porque existe mayor probabilidad de cumplimiento con el tratamiento. Se ha reportado en ENDES $1996\left(^{2}\right)$ que no existen notables diferencias en el porcentaje de niños con anemia según el sexo, el orden de nacimiento, el intervalo de nacimiento, el lugar de residencia y el nivel educativo de la madre, mas sí existe una relacion entre los estados de anemia de las madres y sus niños. Cuando las madres no tienen anemia, un mayor porcentaje de niños tampoco tiene anemia (50\% en comparación con casi $30 \%$ entre los niños cuyas madres tienen anemia severa). Cuando la madre presenta anemia moderada, es también importante la prevalencia de anemia moderada en los niños ( $37 \%$ contra $26 \%$ entre los niños con madres sin anemia) ( $\left.{ }^{2}\right)$.

Se plantea la necesidad de establecer estrategias que incluyan niveles de metas de ejecución para este grupo vulnerable -mujeres en edad fértil, especialmente gestantesya que el estado nutricional pregestacional con déficit en las reservas de hierro también repercute en el niño por na$\operatorname{cer}\left({ }^{17-19}\right)$.

Encontramos en el presente estudio una tasa de incidencia de anernia de $77 \%$, que dista en forma importante de lo encontrado por Zavaleta $\left({ }^{3}\right)$ en $1993(54,6 \%)$ y se acerca más al resultado hallado por Pajuelo-Muñoz en 1996 (66\%).

Más de la mitad de las gestantes (55\%) presentaron una tasa de incidencia acumulada de recuperación (définida como las anémicas que superan el punto de corte $11 \mathrm{~g} / \mathrm{dL}$ al término de un plazo de quince semanas). Asimismo, la respuesta terapéutica de alrededor del $45 \%$ (definida como un incremento igual o mayor a $\mathrm{l} \mathrm{g} / \mathrm{dL}$ en la concentración de hemoglobina) fue debida a la incorporación en las prácticas de las gestantes del consumo de las sales de hierro con vitamina $C$ o con agua, y de dietas ricas en hierro, desplazando los inhibidores de la absorción $\left({ }^{20}\right)$.

Los factores limitantes identificados en el acceso de las madres a un adecuado control prenatal fueron: la información que reciben con respecto a la prevención de anemia y la relación personal entre las madres y el proveedor del servicio. En cuatro estudios financiados por el Programa de Investigación de la Salud Materna de la OMS, se pusieron en evidencia algunas de las razones por las cuales las mujeres no hacían uso de los servicios de salud con los que contaban. A través de los resultados se tuvo que admitir la existencia de impedimentos socioeconómicos y culturales, así como también la necesidad de que los trabajadores de salud dialoguen con las mujeres si lo que se pretende es que las técnicas e instalaciones ofrezcan mayores beneficios para la salud $\left({ }^{18}\right)$.

Al respecto, las promotoras establecieron con las gestantes una estrecha relación de confianza y de comunicación permanente, habiendo de por medio sentimientos de protección social por acompañamiento a lo largo del embarazo, y logrando un cambio en las prácticas de consumo de hierro en $97 \%$ de las gestantes, quienes tomaron regularmente las dosis de hierro planeadas pese a la presentación de algunos efectos colaterales. Existen por otro lado, aspectos culturales que influyen en forma inadecuada, como la "palidez" de la gestante como señal de que el niño se está alimentando bien para ellas, similar a resultados encontrados en una investigación del Ministerio de Salud ( ${ }^{21}$ ), la baja autoestima y la percepción de que el embarazo no necesita cuidados especiales. 
Los fracasos terapéuticos (17\%) estuvieron asociados a la ingesta irregular de las tabletas -sólo una o dos veces por semana-y al consumo de hierro con leche.

Respecto al consumo de sangre animal, experiencias del Centro de Promoción y Estudios en Nutrición, Instituto Nacional de Nutrición y la Universidad Peruana Cayetano Heredia han mostrado que la población peruana considera la sangre animal como un alimento apto para consumo humano en tanto haya higiene en su manipulación. Las evaluaciones de impacto han revelado que el uso de hierro de hemoglobina animal ( ${ }^{12}$ ) "acorta" el tiempo de recuperación de la anemia a uno o dos meses, en contraste con el tratamiento con sales de hierro que requiere de varios meses.

Los materiales editados por el Ministerio de Salud del Perú, según una evaluación interna, adolecen de varias limitaciones, entre otras: a) El $45 \%$ de los mensajes son poco claros, b) El $22 \%$ de los materiales tienen códigos textuales inadecuados para el público objetivo, c) Prioriza materiales impresos, d) Contenidos con tratamiento lineal, expositivo $(22)$.

Una manera de alcanzar elevada eficiencia de los servicios de salud para la atención materna -y con ello reducir la morbilidad y mortalidad materna- es promover una relación dinámica entre el servicio y la comunidad $\left({ }^{18}\right)$. Como se demuestra en esta experiencia, la incorporación de la comunidad organizada, donde las promotoras son los agentes apropiados para ampliar los conocimientos y mejorar las prácticas de las madres con respecto al cuidado de su salud, fue trascendental.

La relación de empatía, de confianza y de gran credibilidad entre las promotoras y las madres favorece la efectividad de la promoción de uso de los servicios de salud.

\section{AGRADECIMIENTOS}

Se agradece a las promotoras de salud de las comunidades de Delicias de Villa y Nueva Esperanza, a los jefes y personal de los centros y puestos de salud de las zonas mencionadas. También al Dr. Luis Benavente por el apoyo prestado, y muy especialmente al Gobierno de Austria -KFSpor su apoyo financiero y material.

\section{BIBLIOGRAFÍA}

1) Downell A, Fernando E, Viteri, Camuerga E. Desnutrición oculta en Latinoamérica: deficiencia de hierro. Centro Asociado de la Facultad de Medicina de la Universidad de El SalvadorCESNI. 1996.

2) Instituto Nacional de Estadística e Informática. Encuesta Demográfica y de Salud Familiar en Perú. ENDES 1996 - In Torme final. INEI. Lima. 1996.

3) Zavaleta N. Prevalencia y determinantes de anemia por deficiencia de hierro en gestantes de Lima Metropolitana. Informe de investigación para OPS, Lima y Libertadores Wari. Oficina Panamericana de la Salud 1993.

4) PRISMA. Informe final Vigilancia Nutricional Región Lima 199192 (Resumen). Asociación Benéfica PRISMA.

5) UN/WHO/FAO: Requerimientos de hierto, Vitamina $A$ y folato. ONU. 1991.

6) Ministerio de Salud. Normas técnicas para la prevención y control de deficiencias de micronutrientes. MINSA. Lima 1998.

7) Trelles J. Mortalidad Perinatal y Riesgo Obstétrico. Anemias un indicador de desnutrición y aumenta el riesgo de mortalidad perinatal. Tesis de Magister en Salud Pública - Universidad Peruana Cayelano Heredia. 1986.

8) Andrade S, Michele G, Shedlin E, Bonilla. Métodos Cualitativos para la evaluación de Programas. USA. The Pathlinder Fund. 1987.

9) Scrimschaw S. Procedimientos de asesoría rápida para programas de nutrición y atención primaria de salud. Universidad de las Naciones Unidas - UNJCEF - UCLA Tokio. 1988.

10) Webb R, Fernandez G. Perú en Námeros 1995. Anuario Estadístico. Cuánto. 1995.

11) OPS - Arroyo L. Mujer, salud y autocuidado. Subregión andina. Perfil del Proyecto 1. Washington D.C. Abril 1992.

12) Benavente L. Mctanálisis experiencias con hierro heme [comunicación personal].

13) FAO. Manejo de proyectos de alimentación y nutrición en comunidades. Guía didáctica. Roma. 1995.

14) Carrasco N. Metodología Educativa para reducir anemia en mujeres. Enfoque y metodología nutricional. CEPREN. Octubre 1997.

15) Aldana C. Para hacer una campaña de comunicación exitosa. La Pizarra Marzo, I(1).

16) PRISMA. Niños. Compendio 1995. Asociación Benéfica PRISMA. Lima 1995.

17) De Meyer EM. The planning and execution of anemia control programme. OMS-Geneve 1989.

18) Maternity services: The need to communicate with the consumer Safe Motherhood News Lelter 1991 Nov-1992 Feb; (7): 9-10.

19) Anemie-les faibles deviennent plus faibles. Maternite Sans Risques 1993 Mar- Jun: (11): 6.

20) Micronutrient supplements help improve infent healt. - Special Feature- Newbom care- Safe Motherhood News Letter 1996; (21): $8-10$.

21) Ministerio de Salud, Proyecto de Salud y Nutrición Básica. Conocimientos, Actitudes y Prácticas en Salud y Nutrición Materno Infantil - Informe de Investigación/No 6. MINSA 1997.

22) Ministerio de Salud. Proyecto de Nutrición Básica. Recopilación y análisis del material comunicacional existente en el ámbito del proyecto salud y nutrición básica. Lima. Julio 1997. 